\title{
ON THE HORN SYSTEM OF PARTIAL DIFFERENTIAL EQUATIONS AND SERIES OF HYPERGEOMETRIC TYPE
}

\author{
T. M. SADYKOV*
}

\begin{abstract}
The paper deals with the Horn system of hypergeometric differential equations. We consider the associated $\mathscr{D}$-module and construct an explicit basis in the space of holomorphic solutions to the Horn system under some assumptions on its parameters.
\end{abstract}

\section{Introduction}

This paper deals with the following system of partial differential equations of hypergeometric type

$$
x_{i} P_{i}\left(x \frac{\partial}{\partial x}\right) y(x)=Q_{i}\left(x \frac{\partial}{\partial x}\right) y(x), \quad i=1, \ldots, n .
$$

Here $P_{i}, Q_{i}$ are nonzero polynomials in $n$ complex variables and

$$
x \frac{\partial}{\partial x}=\left(x_{1} \frac{\partial}{\partial x_{1}}, \ldots, x_{n} \frac{\partial}{\partial x_{n}}\right) .
$$

The system (1) goes back to Horn and Mellin (see [8] and [7], §1.2). It was originally introduced as a natural system of partial differential equations having a given series of hypergeometric type [7] as one of its solutions. In the present paper the system (1) is referred to as the Horn system of hypergeometric differential equations. Here and later we assume that the polynomials $P_{i}, Q_{i}$, $i=1, \ldots, n$, can be represented as products of linear factors. This assumption is used in the proof of Theorem 1.3 which describes solutions to the system of difference equations (3). It also allows one to construct a basis in the space of holomorphic solutions to some systems of the Horn type (Theorem 3.1).

\footnotetext{
* This research was partially supported by the Russian Foundation for Basic Research, grant 9615-96266.

Received June 4, 1999.
} 
Solutions to (1) are closely related to the notion of a Horn series which is defined as a formal (Laurent) series

$$
y(x)=x^{\gamma} \sum_{s \in Z^{n}} \varphi(s) x^{s},
$$

whose coefficients $\varphi(s)$ are characterized by the property that $\varphi\left(s+e_{i}\right)=$ $\varphi(s) R_{i}(s)$ (see [7], §1.2). Here $e_{i}=(0, \ldots, 1, \ldots, 0)$ (1 in the $i$ th position), $R_{i}(s)$ are rational functions. We also use the notations $\gamma=\left(\gamma_{1}, \ldots, \gamma_{n}\right) \in$ $\mathrm{C}^{n}, \operatorname{Re} \gamma_{i} \in[0,1), x^{s}=x_{1}^{s_{1}} \ldots x_{n}^{s_{n}}$.

In the case of two or more variables the Horn system (1) is in general not solvable in the class of series (2) without additional assumptions on the polynomials $P_{i}, Q_{i}$. In section 1 we investigate the solvability of hypergeometric systems of equations. The necessary and sufficient conditions for a formal solution to the system (1) in the class (2) to exist are given in Theorem 1.3.

In section 2 we consider the $\mathscr{D}$-module associated with the Horn system. We give a formula which allows one to compute the dimension of the space of holomorphic solutions to (1) at a generic point under some additional assumptions on the system under study (Theorem 2.8). The author benefited greatly from reading paper [1] by A. Adolphson whose ideas are used in section 2.

In the case of one variable the Horn system (1) coincides with the onedimensional generalized hypergeometric differential equation (see [5], page 77). The construction of a fundamental system of solutions to this equation shows [5] that there exists a basis in its solution space consisting of series (2) if the parameters of the equation are sufficiently general. In section 3 we give a generalization of this fact to the case of systems of partial differential equations of the Horn type (Corollary 3.3). We show that in the case when the polynomial $Q_{i}(s)$ depends on $s_{i}$ only and $\operatorname{deg} Q_{i}(s)>\operatorname{deg} P_{i}(s), i=1, \ldots, n$, there exists a basis in the space of holomorphic solutions to (1) consisting of series (2) if the parameters of the system under study are sufficiently general. This basis is constructed explicitly in section 3 (Theorem 3.1).

The author is very grateful to J. -E. Björk and M. Passare for their constant support and encouragement during the work with this paper. Sincere thanks are due to $\mathrm{C}$. Löfwall for many fruitful discussions which have led to improved proofs of the results in section 2. The author is greatly indebted to A. Tsikh for his comments and advice. 


\section{Solvability conditions, Horn's series and their supports}

Suppose that the series (2) represents a solution to the system (1). Computing the action of the operator $x_{i} P_{i}\left(x \frac{\partial}{\partial x}\right)-Q_{i}\left(x \frac{\partial}{\partial x}\right)$ on this series we arrive at the following system of difference equations

$$
\varphi\left(s+e_{i}\right) Q_{i}\left(s+\gamma+e_{i}\right)=\varphi(s) P_{i}(s+\gamma), \quad i=1, \ldots, n,
$$

where $\left\{e_{i}\right\}_{i=1}^{n}$ is the standard basis of $Z^{n}$. The system (3) is equivalent to (1) as long as we are concerned with those solutions to the Horn system which admit a series expansion of the form (2). Let $Z^{n}+\gamma$ denote the shift in $C^{n}$ of the lattice $Z^{n}$ with respect to the vector $\gamma$. We assume that the polynomials $P_{i}(s), Q_{i}\left(s+e_{i}\right)$ are relatively prime for all $i=1, \ldots, n$. In this section we shall describe nontrivial solutions to the system (3) (i.e. those ones which are not equal to zero identically). While looking for a solution to (3) which is different from zero on some subset $S$ of $Z^{n}$ we shall assume that the polynomials $P_{i}(s), Q_{i}(s)$, the set $S$ and the vector $\gamma$ satisfy the condition

$$
\left|P_{i}(s+\gamma)\right|+\left|Q_{i}\left(s+\gamma+e_{i}\right)\right| \neq 0,
$$

for any $s \in S$ and for all $i=1, \ldots, n$. That is, for any $s \in S$ the equality $P_{i}(s+\gamma)=0$ implies that $Q_{i}\left(s+\gamma+e_{i}\right) \neq 0$ and $Q_{i}\left(s+\gamma+e_{i}\right)=0$ implies $P_{i}(s+\gamma) \neq 0$. This assumption eliminates the case when a solution to (3) can independently take arbitrary values at two adjacent points in the set $S$.

The system (3) can be considered for $s$ varying over $C^{n}$ as well as for $s$ being a discrete argument, that is, for $s \in Z^{n}$. In the first case the solution to (3) is determined uniquely up to a periodic factor. The following Theorem (see [10]) gives necessary and sufficient conditions for a nontrivial solution to the system (3) to exist in the case when $s \in \mathrm{C}^{n}$.

THEOREM 1.1. The system (3) has a nontrivial solution if and only if the conditions

$$
R_{i}\left(s+e_{j}\right) R_{j}(s)=R_{j}\left(s+e_{i}\right) R_{i}(s)
$$

hold for all $i, j=1, \ldots, n$. Here $R_{i}(s)=P_{i}(s) / Q_{i}\left(s+e_{i}\right)$. A solution to a solvable system (3) is determined uniquely up to a factor $\phi(s)$ satisfying the periodicity conditions $\phi\left(s+e_{i}\right) \equiv \phi(s), i=1, \ldots, n$.

In the paper [10] an algorithm for constructing the general solution to a solvable system (3) was developed. It was shown that in accordance with the 
Ore-Sato theorem [12] (see also [7], §1.2) it must be of the form (6)

$\varphi_{0}(s)=t_{1}^{s_{1}} \ldots t_{n}^{s_{n}} \prod_{i=1}^{p} \Gamma\left(\left\langle A_{i}, s+\gamma\right\rangle-c_{i}\right)\left(\prod_{i=p+1}^{q} \Gamma\left(\left\langle A_{i}, s+\gamma\right\rangle-c_{i}\right)\right)^{-1} \phi(s)$.

Here $\Gamma(z)$ is the Euler $\Gamma$-function, $t_{i} \in \mathrm{C}, A_{i}=\left(A_{i 1}, \ldots, A_{i n}\right) \in \mathrm{Z}^{n}, p, q \in$ $\mathrm{N}_{0}, p \leq q$, and $\phi(s)$ is an arbitrary periodic function satisfying $\phi\left(s+e_{i}\right) \equiv$ $\phi(s), i=1, \ldots, n$. The vectors $A_{i}$ correspond to the linear factors of the polynomials $P_{i}(s+\gamma), Q_{i}\left(s+\gamma+e_{i}\right)$. Notice that the function $\varphi_{0}(s)$ is given by the product of a meromorphic function and a periodic function and therefore does not need to give rise to a solution depending on a discrete argument. Indeed, $\varphi_{0}(s)$ may have poles or zeros at some points of the lattice. We shall show in this section how to choose the periodic factor $\phi(s)$ in order to obtain a solution to (3) depending on a discrete argument.

We assume throughout this paper that the polynomials $P_{i}(s), Q_{i}(s)$ satisfy the compatibility conditions (5). In the case when $s \in Z^{n}$ these conditions remain necessary for the solvability of (3) on a set where the coefficients of the equations (3) do not vanish (see also Remark 1.5). Yet, in this case they are neither sufficient for (3) to be solvable nor guarantee uniqueness of its solution. The reason for this is that under natural assumptions (given in Theorem 1.3) on the polynomials $P_{i}(s), Q_{i}(s)$ a solution to (3) can be defined to be zero outside some subset of the lattice $Z^{n}$. This cut-off function can be shown to satisfy the system (3) and therefore provides a solution of a new type. This essential difference between the case when $s \in \mathrm{Z}^{n}$ and the case of a continuous argument is illustrated by the following example.

Example 1.2. Consider the following system of the Horn type

$$
\left\{\begin{array}{l}
x_{1}\left(x_{1} \frac{\partial}{\partial x_{1}}+x_{2} \frac{\partial}{\partial x_{2}}-1\right) y(x)=\left(x_{1} \frac{\partial}{\partial x_{1}}\right) y(x), \\
x_{2}\left(x_{1} \frac{\partial}{\partial x_{1}}+x_{2} \frac{\partial}{\partial x_{2}}-1\right) y(x)=\left(x_{2} \frac{\partial}{\partial x_{2}}\right) y(x) .
\end{array}\right.
$$

Let $\gamma=0$ and consider the corresponding system of difference equations

$$
\left\{\begin{array}{l}
\varphi\left(s+e_{1}\right)\left(s_{1}+1\right)=\varphi(s)\left(s_{1}+s_{2}-1\right), \\
\varphi\left(s+e_{2}\right)\left(s_{2}+1\right)=\varphi(s)\left(s_{1}+s_{2}-1\right) .
\end{array}\right.
$$

This system of difference equations is easily seen to satisfy the compatibility conditions (5). By Theorem 1.1 its general solution is given by the product of 
a periodic function $\phi(s)$ and the function

$$
\varphi_{1}(s)=\Gamma\left(s_{1}+s_{2}-1\right) /\left(\Gamma\left(s_{1}+1\right) \Gamma\left(s_{2}+1\right)\right) .
$$

Thus, for $s$ varying over $\mathrm{C}^{2}$, a solution to (8) is given by a function which is different from zero almost everywhere. Yet, the only way of constructing a nontrivial solution to (8) in the case when $s$ is considered as a discrete argument varying over $Z^{2}$, is to set $\varphi(s)$ to be zero everywhere except for the finite set consisting of three points $(0,0),(1,0),(0,1)$. Choosing $\phi(s)=(-1)^{s_{1}+s_{2}} \sin \left(\pi\left(s_{1}+s_{2}-1\right)\right) / \pi$ and using the Euler completion formula $\Gamma(z) \Gamma(1-z)=\pi / \sin (\pi z)$ we obtain a solution $\varphi_{2}(s)$ to the system $(8)$ which is well-defined on this set: $\varphi_{2}(s)=(-1)^{s_{1}+s_{2}} /\left(\Gamma\left(2-s_{1}-s_{2}\right) \Gamma\left(s_{1}+1\right) \Gamma\left(s_{2}+1\right)\right)$. Thus the solution $\varphi(s)$ to (8) for $s \in Z^{2}$ is given by $\varphi(0,0)=\varphi_{2}(0,0)=1$, $\varphi(1,0)=\varphi_{2}(1,0)=-1, \varphi(0,1)=\varphi_{2}(0,1)=-1$, and $\varphi(s)=0$ on the rest of $Z^{2}$. Theorem 1.3 which will be proved later in this section yields that no other choice of the function $\varphi(s)$ is possible and therefore the only solution to the system (7), which can be represented as a series (2), is given by $y(x)=c\left(1-x_{1}-x_{2}\right)$. Here $c$ is an arbitrary constant. Moreover, the function $y(x)=1-x_{1}-x_{2}$ gives a basis in the space of holomorphic solutions to (7).

Let $\varphi(s)$ be a solution to (3) for $s \in Z^{n}$. We define the support of $\varphi(s)$ to be the subset of the lattice $Z^{n}$ where $\varphi(s)$ is different from zero. The support $S$ of a solution to (3) is called irreducible if there exists no solution to (3) supported in a proper nonempty subset of $S$. A set $S \subset Z^{n}$ is called $Z^{n}$-convex if the inclusion $\left\{\lambda s^{(0)}+(1-\lambda) s^{(1)}: \lambda \in[0,1]\right\} \cap Z^{n} \subset S$ holds for any $s^{(0)}, s^{(1)} \in S$. A set $S \subset Z^{n}$ is said to be $Z^{n}$-connected if any two points of $S$ can be connected by a polygonal line with unit sides and vertices in $S$. Any irreducible support is a $Z^{n}$ connected set. If the conditions (4) are satisfied then any $Z^{n}$-connected support is irreducible. A formal series $x^{\gamma} \sum_{s \in Z^{n}} \varphi(s) x^{s}$ is called a formal solution to the system (1) if the function $\varphi(s)$ satisfies the equations (3) at each point of the lattice $Z^{n}$. The following Theorem gives necessary and sufficient conditions for a solution to the system (3) supported in some set $S \subset Z^{n}$ to exist.

THEOREM 1.3. For $S \subset Z^{n}$ define

$$
S_{i}^{\prime}=\left\{s \in S: s+e_{i} \notin S\right\}, \quad S_{i}^{\prime \prime}=\left\{s \notin S: s+e_{i} \in S\right\}, \quad i=1, \ldots, n .
$$

Suppose that the conditions (4) are satisfied on $S$. Then there exists a solution to the system (3) supported in $S$ if and only if the following conditions are fulfilled:

$$
\begin{array}{rlr}
\left.P_{i}(s+\gamma)\right|_{S_{i}^{\prime}}=0, & \left.Q_{i}\left(s+\gamma+e_{i}\right)\right|_{S_{i}^{\prime \prime}}=0, & i=1, \ldots, n, \\
\left.P_{i}(s+\gamma)\right|_{S \backslash S_{i}^{\prime}} \neq 0, & \left.Q_{i}\left(s+\gamma+e_{i}\right)\right|_{S} \neq 0, & i=1, \ldots, n .
\end{array}
$$


If, in addition, the constants $c_{i}$ in (6) satisfy the condition that $c_{i}-c_{j} \notin \mathrm{Z}$ for $i \neq j$ then all irreducible supports of solutions to (3) are $Z^{n}$-convex sets.

REMARK 1.4. Without loss of generality we may assume that the set $S$ is $Z^{n}$ connected. For otherwise we may divide the set $S$ into two subsets which are not connected through the equations (3) and consider these subsets separately.

Remark 1.5. In the case of a discrete argument the conditions (5) may be violated for a solvable system (3) if there exists a sufficiently small subset of the lattice satisfying the conditions of Theorem 1.3. For instance, let $n=2$ and consider the system of difference equations

$$
\left\{\begin{array}{l}
\varphi\left(s+e_{1}\right) s_{1}=\varphi(s)\left(s_{1}+s_{2}-2\right)\left(s_{1}+s_{2}-3\right), \\
\varphi\left(s+e_{2}\right) s_{2}=\varphi(s)\left(s_{1}+s_{2}-2\right) .
\end{array}\right.
$$

The function $\varphi(s)$ which equals 1 at the point $(1,1)$ and zero on the rest of the lattice solves the system (11) though the compatibility conditions (5) are not satisfied in this example.

Proof of Theorem 1.3. Necessity. Let $\varphi(s)$ be a solution to (3), $S=$ $\operatorname{supp} \varphi$. Let $s^{(0)} \in S_{i}^{\prime}$. Since $\varphi\left(s^{(0)}\right) \neq 0$ and $\varphi\left(s^{(0)}+e_{i}\right)=0$, it follows from the $i$ th equation of (3) that $P_{i}\left(s^{(0)}+\gamma\right)=0$. Analogously, if $s^{(0)} \in S_{i}^{\prime \prime}$ then $\varphi\left(s^{(0)}\right)=0, \varphi\left(s^{(0)}+e_{i}\right) \neq 0$, which yields $Q_{i}\left(s^{(0)}+\gamma+e_{i}\right)=0$. This proves the necessity of the conditions (9) for the system (3) to be solvable. To show the necessity of (10) we assume that $P_{i}\left(s^{(0)}+\gamma\right)=0$ for $s^{(0)} \in S$. Then the $i$ th equation of (3) together with (4) gives $\varphi\left(s^{(0)}+e_{i}\right)=0$, which means that $s^{(0)} \in S_{i}^{\prime}$. Next, if $Q_{i}\left(s^{(0)}+\gamma+e_{i}\right)=0$ then it follows from (3) and (4) that $\varphi\left(s^{(0)}\right)=0$, that is, $s^{(0)} \notin S$.

Sufficiency. We shall construct a function $\varphi_{S}(s)$ satisfying (3) and supported in $S$. By Remark 1.4 we may assume that the set $S$ is $Z^{n}$-connected. Choose an arbitrary point $s^{(0)} \in S$ and set $\varphi_{S}\left(s^{(0)}\right)=1$. The equations (3) may be viewed as recurrent relations which allow one to compute the value of $\varphi_{S}\left(s^{(0)} \pm e_{i}\right)$ for any $i=1, \ldots, n$ unless $P_{i}\left(s^{(0)}+\gamma\right)=0$ or $Q_{i}\left(s^{(0)}+\right.$ $\left.\gamma+e_{i}\right)=0$. Repeating this argument, we can compute the value of $\varphi_{S}(s)$ at any point $s^{(1)} \in S$ which may be connected with $s^{(0)}$ by a polygonal line with unit segments and vertices in $S$. By our assumption on the set $S$ we can define $\varphi_{S}(s)$ for any $s \in S$ since by (10) the polynomial $Q_{i}\left(s+\gamma+e_{i}\right)$ does not vanish on $S$ for any $i=1, \ldots, n$ and since the polynomial $P_{i}(s+\gamma)$ vanishes on $S_{i}^{\prime}$ only. The function $\varphi_{S}(s)$ is well-defined since the compatibility conditions (5) are fulfilled. (These conditions imply that the value of $\varphi_{S}(s)$ at a point $s^{(1)} \in S$ obtained by iterating the equations (3) does not depend on the path connecting $s^{(0)}$ and $s^{(1)}$.) 
Let us define $\varphi_{S}(s)$ to be zero outside $S$. The function $\varphi_{S}(s)$ satisfies the equations (3) on $Z^{n} \backslash\left(\bigcup_{i=1}^{n}\left(S_{i}^{\prime} \cup S_{i}^{\prime \prime}\right)\right)$ by the construction. This follows since $\varphi(s)=\varphi\left(s+e_{i}\right)=0$ on $Z^{n} \backslash\left(S \cup\left(\bigcup_{i=1}^{n} S_{i}^{\prime \prime}\right)\right)$, for any $i=1, \ldots, n$, and since $\varphi(s)$ was defined through the equations (3) on $S \backslash\left(\bigcup_{i=1}^{n} S_{i}^{\prime}\right)$. The conditions (9) yield that these equations are also satisfied on $\bigcup_{i=1}^{n}\left(S_{i}^{\prime} \cup S_{i}^{\prime \prime}\right)$, which shows that the conditions (9),(10) are sufficient for a solution to (3) supported in $S$ to exist.

Suppose now that $S \subset Z^{n}$ is the irreducible support of some solution to (3). The conditions (4) imply that $S$ is a $Z^{n}$-connected set. Let $s^{(0)}, s^{(1)} \in S$ and let $L$ be a polygonal line with unit sides and vertices in $S$ connecting $s^{(0)}$ and $s^{(1)}$. Without loss of generality we may assume that $s_{1}^{(0)}-s_{1}^{(1)}>0$. Suppose $S$ is not $Z^{n}$-convex. Then there exists a point $s^{(2)} \in S_{1}^{\prime} \cup S_{1}^{\prime \prime}$ such that any hyperplane $\langle B, s+\gamma\rangle-d=0$ with $B_{1} \neq 0$ through the point $s^{(2)}$ intersects $L$. Suppose that $s^{(2)} \in S_{1}^{\prime \prime}$. The condition (9) gives $Q_{1}\left(s^{(2)}+\gamma+e_{1}\right)=0$. The construction of the function (6) (see [10]) yields that there exists $i_{0} \in\{1, \ldots, q\}$ such that $\left\langle A_{i_{0}}, s^{(2)}+\gamma+e_{1}\right\rangle-c_{i_{0}}=0$. It follows from Lemma 1 in [10] that the vector $A_{i_{0}}$ can be chosen so that $A_{i_{0}}>0$. Since $c_{i}-c_{j} \notin \mathrm{Z}$ for $i \neq j$ by the assumption, it follows from (9) and (5) that the polynomial $Q_{1}(s)$ is divisible by the product $\tilde{Q}_{1}(s)=\prod_{k=0}^{A_{i_{1}}-1}\left(\left\langle A_{i_{0}}, s\right\rangle+k-c_{i_{0}}\right)$. Thus the set $Z^{n} \cap L \cap\left\{s: Q_{1}\left(s+\gamma+e_{1}\right)=0\right\}$ is nonempty which is impossible by (10). Similar arguments show that $s^{(2)} \in S_{1}^{\prime}$ is also impossible which proves $Z^{n}$ convexity of an irreducible support. The proof is complete.

Theorem 1.3 yields the existence of a solution to (3) supported in a set $S \subset$ $Z^{n}$ which satisfies the conditions (4), (5), (9) and (10). Let us now explain how one can construct this solution explicitly under some additional assumptions. It suffices to consider the case when $S$ is $Z^{n}$-connected. We show how the periodic function $\phi(s)$ in (6) can be chosen in order to make $\varphi_{0}(s)$ well-defined on $S$. Since $S$ is assumed to be $Z^{n}$-connected it follows by (4) and Theorem 1.3 that $S$ is $Z^{n}$-convex. Let $\tilde{S}$ denote the convex hull of the set $S$. Assume that none of the polynomials $P_{1}(s+\gamma), \ldots, P_{n}(s+\gamma), Q_{1}\left(s+\gamma+e_{1}\right), \ldots, Q_{n}\left(s+\gamma+e_{n}\right)$ vanishes in the interior of the convex set $\tilde{S}$. Let $A_{i_{1}}, \ldots, A_{i_{r}} \in\left\{A_{1}, \ldots, A_{q}\right\}$ be the vectors introduced in (6) which are characterized by the property that the set $\tilde{S}$ lies in the half-space defined by $\left\langle A_{i_{j}}, s+\gamma\right\rangle-c_{i_{j}} \leq 0, j=1, \ldots, r$. Choose

$$
\phi(s)=\prod_{j=1}^{r}\left((-1)^{\left(\left\langle A_{i_{j}}, s+\gamma\right\rangle-c_{i_{j}}\right)} \frac{1}{\pi} \sin \pi\left(\left\langle A_{i_{j}}, s+\gamma\right\rangle-c_{i_{j}}\right)\right)^{\varepsilon_{j}},
$$

where $\varepsilon_{j}=1$ for $1 \leq i_{j} \leq p$ and $\varepsilon_{j}=-1$ for $p<i_{j} \leq q$. Since $A_{j} \in \mathrm{Z}^{n}$ it follows that $\phi(s)$ defined in (12) is periodic in $s_{1}, \ldots, s_{n}$ with the period 1. 
Applying the Euler completion formula $\Gamma(z) \Gamma(1-z)=\pi / \sin \pi z$ we see that the function $\varphi_{0}(s)$ in (6) with the periodic factor $\phi(s)$ given by (12) is well-defined on $S$. Let $\varphi_{S}(s)$ be equal to $\varphi_{0}(s)$ for $s \in S$ and let it be zero otherwise. Then the equations (3) are satisfied everywhere in $Z^{n} \backslash\left(S \cup\left(\bigcup_{i=1}^{n} S_{i}^{\prime \prime}\right)\right)$ since $\varphi_{S}(s)=\varphi_{S}\left(s+e_{i}\right)=0$ on this set, for any $i=1, \ldots, n$. These equations are also satisfied on $S \backslash \bigcup_{i=1}^{n} S_{i}^{\prime}$ by the construction of $\varphi_{S}(s)$. Finally, the equations (3) are satisfied on $\bigcup_{i=1}^{n}\left(S_{i}^{\prime} \cup S_{i}^{\prime \prime}\right)$ by (9) and (10). Thus the constructed function $\varphi_{S}(s)$ is indeed a solution to (3). The formulas (6) and (12) are used in examples 2 and 3 for constructing explicit solutions to some systems of the Horn type.

Notice that the number of linearly independent solutions to the system (1) in the class of series (2) can be smaller than the number of irreducible supports of solutions to the associated system of difference equations (3). A simple example is given by the ordinary differential equation $x\left(x \frac{d}{d x}-a\right) y(x)=$ $\left(x \frac{d}{d x}-b\right) y(x)$, where $a, b \in \mathrm{Z}, a<b$. There are two disjoint subsets of the lattice Z which satisfy the conditions of Theorem 1.3, namely $S_{1}=\{s \in$ $Z, s \geq b\}$ and $S_{2}=\{s \in Z, s \leq a\}$. Yet, the corresponding solutions $y_{1}(x)=$ $\sum_{n=b}^{\infty} \frac{\Gamma(n-a)}{\Gamma(n-b+1)} x^{n}$ and $y_{2}(x)=(-1)^{b-a} \sum_{n=-\infty}^{a} \frac{\Gamma(b-n)}{\Gamma(a+1-n)} x^{n}$ are essentially the same since they represent the same analytic function in the domains $\{|x|<1\}$ and $\{|x|>1\}$ respectively. For more examples of this type see section 3 .

Theorem 1.3 will be used in section 3 for constructing an explicit basis in the space of holomorphic solutions to the Horn system in the case when $\operatorname{deg} Q_{i}>\operatorname{deg} P_{i}$ and the polynomial $Q_{i}(s)$ depends on $s_{i}$ only, $i=1, \ldots, n$. In the next section we compute the dimension of the space of holomorphic solutions to (1) at a generic point.

\section{The $\mathscr{D}$-module associated with the Horn system}

Let $G_{i}$ denote the differential operator $x_{i} P_{i}\left(x \frac{\partial}{\partial x}\right)-Q_{i}\left(x \frac{\partial}{\partial x}\right), i=1, \ldots, n$. Let $\mathscr{D}$ be the Weyl algebra in $n$ variables [3], and define $\mathscr{M}=\mathscr{D} / \sum_{i=1}^{n} \mathscr{D} G_{i}$ to be the left $\mathscr{D}$-module associated with the system (1). Let $R=\mathrm{C}\left[z_{1}, \ldots, z_{n}\right]$ and $R[x]=R\left[x_{1}, \ldots, x_{n}\right]=\mathrm{C}\left[x_{1}, \ldots, x_{n}, z_{1}, \ldots, z_{n}\right]$. We make $R[x]$ into a left $\mathscr{D}$-module by defining the action of $\partial_{j}$ on $R[x]$ by

$$
\partial_{j}=\frac{\partial}{\partial x_{j}}+z_{j}
$$

Let $\Phi: \mathscr{D} \rightarrow R[x]$ be the $\mathscr{D}$-linear map defined by

$$
\Phi\left(x_{1}^{a_{1}} \ldots x_{n}^{a_{n}} \partial_{1}^{b_{1}} \ldots \partial_{n}^{b_{n}}\right)=x_{1}^{a_{1}} \ldots x_{n}^{a_{n}} z_{1}^{b_{1}} \ldots z_{n}^{b_{n}} .
$$

It is easily checked that $\Phi$ is an isomorphism of $\mathscr{D}$-modules. In this section we establish some properties of linear operators acting on $R[x]$. We aim to 
construct a commutative family of $\mathscr{D}$-linear operators $W_{i}: R[x] \rightarrow R[x]$, $i=1, \ldots, n$ which satisfy the equality $\Phi\left(G_{i}\right)=W_{i}(1)$. The crucial point which requires additional assumptions on the parameters of the system (1) is the commutativity of the family $\left\{W_{i}\right\}_{i=1}^{n}$ which is needed for computing the dimension (as a C-vector space) of the module $R[x] / \sum_{i=1}^{n} W_{i} R[x]$ at a fixed point $x^{(0)}$. We construct the operators $W_{i}$ and show that they commute with one another under some additional assumptions on the polynomials $Q_{i}(s)$ (Lemma 2.5). However, no additional assumptions on the polynomials $P_{i}(s)$ are needed as long as the compatibility conditions (5) are fulfilled.

Following the spirit of Adolphson [1] we define operators $D_{i}: R[x] \rightarrow$ $R[x]$ by setting

$$
D_{i}=z_{i} \frac{\partial}{\partial z_{i}}+x_{i} z_{i}, i=1, \ldots, n .
$$

It was pointed out in [1] that the operators (15) form a commutative family of $\mathscr{D}$-linear operators. Let $D$ denote the vector $\left(D_{1}, \ldots, D_{n}\right)$. For any $i=$ $1, \ldots, n$ we define operator $\nabla_{i}: R[x] \rightarrow R[x]$ by $\nabla_{i}=z_{i}^{-1} D_{i}$. This operator commutes with the operators $\partial_{j}$ since both $D_{i}$ and the multiplication by $z_{i}^{-1}$ commute with $\partial_{j}$. Moreover, the operator $\nabla_{i}$ commutes with $\nabla_{j}$ for all $1 \leq$ $i, j \leq n$ and with $D_{j}$ for $i \neq j$. In the case $i=j$ we have $\nabla_{i} D_{i}=\nabla_{i}+D_{i} \nabla_{i}$.

REMARK 2.1. A power of the operator $x_{i} \frac{\partial}{\partial x_{i}}$ admits the following expansion: $\left(x_{i} \frac{\partial}{\partial x_{i}}\right)^{k}=\sum_{j=1}^{k} S_{j, k} x_{i}^{j} \frac{\partial^{j}}{\partial x_{i}^{j}}$, where $S_{j, k}$ are the Stirling numbers of the second kind (see [2], page 89). This allows one to determine the constants $h_{\alpha}^{(i)}$ in the expansion of the operator $x_{i} P_{i}\left(x \frac{\partial}{\partial x}\right)-Q_{i}\left(x \frac{\partial}{\partial x}\right)=\sum_{\alpha} h_{\alpha}^{(i)} x^{\beta_{\alpha}^{(i)}} \frac{\partial^{|\alpha|}}{\partial x^{\alpha}}$.

The following lemma (which can be applied to a more general family of differential operators than $\left\{G_{i}\right\}_{i=1}^{n}$ ) gives $\mathscr{D}$-linear operators $W_{i}: R[x] \rightarrow$ $R[x]$ which satisfy $\Phi\left(G_{i}\right)=W_{i}(1)$.

Lemma 2.2. Let $m_{1}, \ldots, m_{n} \in \mathrm{N}_{0}$ and let $V_{\alpha}^{(i)},|\alpha| \leq m_{i}$ be polynomials in $n$ variables. Consider the family of generalized hypergeometric operators

$$
\tilde{G}_{i}=\sum_{|\alpha| \leq m_{i}} x^{\alpha} V_{\alpha}^{(i)}\left(x \frac{\partial}{\partial x}\right), i=1, \ldots, n .
$$

Let us define operators $\tilde{W}_{i}, i=1, \ldots, n$ by

$$
\tilde{W}_{i}=\sum_{|\alpha| \leq m_{i}} V_{\alpha}^{(i)}(D) \nabla^{\alpha}
$$

Then $\tilde{W}_{i}$ is a $\mathscr{D}$-linear operator on $R[x]$ satisfying $\Phi\left(\tilde{G}_{i}\right)=\tilde{W}_{i}(1)$. 
Proof. The $\mathscr{D}$-linearity of $\tilde{W}_{i}$ follows since the operators $D_{i}$ and $\nabla_{i}$ are $\mathscr{D}$-linear, for all $i, j=1, \ldots, n$. Thus we need to show that for any $\alpha, \beta \in \mathbb{N}_{0}^{n}$

$$
\left(D_{1}^{\alpha_{1}} \ldots D_{n}^{\alpha_{n}} \nabla_{1}^{\beta_{1}} \ldots \nabla_{n}^{\beta_{n}}\right)(1)=\Phi\left(x^{\beta}\left(x_{1} \frac{\partial}{\partial x_{1}}\right)^{\alpha_{1}} \ldots\left(x_{n} \frac{\partial}{\partial x_{n}}\right)^{\alpha_{n}}\right)
$$

and that

$$
\left(D_{1}^{\alpha_{1}} \ldots D_{n}^{\alpha_{n}}\right)(1)=\Phi\left(\left(x_{1} \frac{\partial}{\partial x_{1}}\right)^{\alpha_{1}} \ldots\left(x_{n} \frac{\partial}{\partial x_{n}}\right)^{\alpha_{n}}\right) .
$$

The equation (16) follows from (17) since $D_{i} \nabla_{j}(1)=D_{i}\left(x_{j}\right)=x_{j} D_{i}(1)$ and $\Phi\left(x_{j} F\right)=x_{j} \Phi(F)$ for any differential operator $F \in \mathscr{D}$. For proving (17) we notice that $D_{i}^{\alpha_{i}}(1)$ can be written in the form $D_{i}^{\alpha_{i}}(1)=\sum_{k=1}^{\alpha_{i}} c_{k, \alpha_{i}} x_{i}^{k} z_{i}^{k}$. Since $D_{i}(1)=x_{i} z_{i}$ and

$$
D_{i}^{k+1}(1)=c_{1, k} x_{i} z_{i}+\sum_{j=2}^{k}\left(j c_{j, k}+c_{j-1, k}\right) x_{i}^{j} z_{i}^{j}+x_{i}^{k+1} z_{i}^{k+1}
$$

it follows that the constants $c_{j, k}$ are the Stirling numbers $S_{j, k}$ of the second kind as in Remark 2.1. Indeed, they are determined by the same recurrent relation with the same initial condition as $S_{j, k}$. Thus we get $D_{i}^{\alpha_{i}}(1)=\sum_{k=1}^{\alpha_{i}} S_{k, \alpha_{i}} x_{i}^{k} z_{i}^{k}$ and Remark 2.1 gives $D_{i}^{\alpha_{i}}(1)=\Phi\left(\left(x_{i} \frac{\partial}{\partial x_{i}}\right)^{\alpha_{i}}\right)$. The equality (17) follows now from the identities

$$
\begin{aligned}
D_{1}^{\alpha_{1}} \ldots D_{n}^{\alpha_{n}}(1) & =\prod_{i=1}^{n} D_{i}^{\alpha_{i}}(1), \\
\Phi\left(\left(x_{1} \frac{\partial}{\partial x_{1}}\right)^{\alpha_{1}} \ldots\left(x_{n} \frac{\partial}{\partial x_{n}}\right)^{\alpha_{n}}\right) & =\prod_{i=1}^{n} \Phi\left(\left(x_{i} \frac{\partial}{\partial x_{i}}\right)^{\alpha_{i}}\right),
\end{aligned}
$$

which hold for any $\alpha \in \mathbb{N}_{0}^{n}$. The proof is complete.

Thanks to Lemma 2.2 we may define operators

$$
W_{i}=P_{i}(D) \nabla_{i}-Q_{i}(D)
$$

such that for any $i=1, \ldots, n, W_{i}$ is a $\mathscr{D}$-linear operator satisfying the identity $\Phi\left(G_{i}\right)=W_{i}(1)$.

REMARK 2.3. Let $F_{i}=\sum_{\alpha} c_{\alpha}^{(i)} x^{\beta_{\alpha}^{(i)}}\left(\frac{\partial}{\partial x}\right)^{\alpha}, i=1, \ldots, n$ be a family of differential operators such that $\beta_{\alpha}^{(i)} \geq \alpha$ for every $\alpha$ which is present in the sum. Here $\alpha, \beta_{\alpha}^{(i)} \in \mathrm{N}_{0}^{n}$. The existence of a family of $\mathscr{D}$-linear operators $\left\{\tilde{W}_{i}\right\}_{i=1}^{n}$ 
satisfying $\Phi\left(F_{i}\right)=\tilde{W}_{i}(1)$ (which follows from Lemma 2.2) can be also proved in the following way. Let $x^{k} z^{q} \in R[x]$ be an arbitrary monomial satisfying $q_{i} \leq k_{i}, i=1, \ldots, n$. For each $j=1, \ldots, n$ define

$$
T_{j}:=\left(\prod_{k=0}^{q_{j}-1}\left(D_{j}-k\right)\right)\left(\nabla_{j}\right)^{k_{j}-q_{j}} .
$$

Notice that the operators $T_{1}, \ldots, T_{n}$ commute. Let $T$ be the product of $T_{1}, \ldots$, $T_{n}$, then $T(1)=x^{k} z^{q}$. Thus the existence of the desired family of operators follows by $\mathscr{D}$-linearity of $T_{j}$. The identity

$$
\begin{aligned}
& \left(D_{j}-\left(q_{j}-1\right)\right) \ldots\left(D_{j}-1\right) D_{j}\left(x_{j}^{\mu} z_{j}^{\lambda}\right) \\
& \quad=\sum_{k=0}^{q_{j}-1}\left(\begin{array}{c}
q_{j} \\
k
\end{array}\right) \lambda(\lambda-1) \ldots\left(\lambda-\left(q_{j}-1-k\right)\right) x_{j}^{\mu+k} z_{j}^{\lambda+k}+x_{j}^{\mu+q_{j}} z_{j}^{\lambda+q_{j}},
\end{aligned}
$$

which can be easily verified by induction, allows one to compute the action of the operator $T_{j}$ on an arbitrary element of $R[x]$.

It follows by the $\mathscr{D}$-linearity of $W_{i}$ that $\sum_{i=1}^{n} W_{i} R[x]$ and $R[x] / \sum_{i=1}^{n} W_{i} R[x]$ can be considered as left $\mathscr{D}$-modules. The next argument which is due to Adolphson [1] shows that the $\mathscr{D}$-module $\mathscr{M}$, associated with the Horn system (1), is isomorphic to $R[x] / \sum_{i=1}^{n} W_{i} R[x]$.

THEOREM 2.4. The following isomorphism holds true:

$$
\mathscr{M} \simeq R[x] /\left(\sum_{j=1}^{n} W_{j} R[x]\right) .
$$

Proof. It follows by $\mathscr{D}$-linearity of the operators $W_{i}$ that the sum $\sum_{j=1}^{n} \mathscr{D} G_{j}$ belongs to the kernel of the map

$$
\mathscr{D} \rightarrow R[x] /\left(\sum_{j=1}^{n} W_{j} R[x]\right),
$$

induced by the isomorphism of $\mathscr{D}$-modules $\Phi: \mathscr{D} \rightarrow R[x]$. To show that this sum is equal to the kernel it suffices to prove that for any $b=\left(b_{1}, \ldots, b_{n}\right) \in \mathrm{N}_{0}^{n}$, and any $u_{b}(x) \in \mathrm{C}[x]$ there exists $\xi \in \mathscr{D} W_{j}$ such that $\Phi(\xi)=W_{j}\left(u_{b}(x) z^{b}\right)$. Let us define $\xi$ by $\xi=u_{b}(x) \prod_{i=1}^{n}\left(\frac{\partial}{\partial x_{i}}\right)^{b_{i}} G_{j}$. Using $\mathscr{D}$-linearity of $W_{j}$ and the 
equality $\Phi\left(G_{i}\right)=W_{i}(1)$ we obtain

$$
\begin{aligned}
\Phi\left(u_{b}(x) \prod_{i=1}^{n}\left(\frac{\partial}{\partial x_{i}}\right)^{b_{i}} G_{j}\right) & =u_{b}(x) \prod_{i=1}^{n} \partial_{i}^{b_{i}} \Phi\left(G_{j}\right) \\
& =u_{b}(x) \prod_{i=1}^{n}\left(\frac{\partial}{\partial x_{i}}+z_{i}\right)^{b_{i}} W_{j}(1) \\
& =W_{j}\left(u_{b}(x) \prod_{i=1}^{n}\left(\frac{\partial}{\partial x_{i}}+z_{i}\right)^{b_{i}}(1)\right) \\
& =W_{j}\left(u_{b}(x) z^{b}\right) .
\end{aligned}
$$

This shows that $\sum_{j=1}^{n} \mathscr{D} G_{j}$ coincides with the kernel of the map (19) and completes the proof of the Theorem.

In the general case the operators $W_{i}=P_{i}(D) \nabla_{i}-Q_{i}(D)$ do not commute since $D_{i}$ does not commute with $\nabla_{i}$. However, this family of operators may be shown to be commutative under some assumptions on the polynomials $Q_{i}(s)$ in the case when the polynomials $P_{i}(s), Q_{i}(s)$ satisfy the compatibility conditions (5). The following Lemma holds.

Lemma 2.5. The operators $W_{i}=P_{i}(D) \nabla_{i}-Q_{i}(D)$ commute with one another if and only if the polynomials $P_{i}(s), Q_{i}(s)$ satisfy the compatibility conditions (5) and for each $i=1, \ldots, n$ the polynomial $Q_{i}\left(s_{1}, \ldots, s_{n}\right)$ depends on $s_{i}$ only.

Proof. Since $\nabla_{i}=z_{i}^{-1}+D_{i} z_{i}^{-1}$ it follows that $\nabla_{i} D_{i}=\nabla_{i}+D_{i} \nabla_{i}$ and that $\nabla_{i}$ commutes with $D_{j}$ for $i \neq j$. Hence for any $\alpha=\left(\alpha_{1}, \ldots, \alpha_{n}\right) \in \mathrm{N}_{0}^{n}$

$$
\nabla_{i} D_{1}^{\alpha_{1}} \ldots D_{n}^{\alpha_{n}}=D_{1}^{\alpha_{1}} \ldots\left(D_{i}+1\right)^{\alpha_{i}} \ldots D_{n}^{\alpha_{n}} \nabla_{i} .
$$

Let $E_{i}^{t}$ denote the operator which increases the $i$ th argument by $t$, that is, $E_{i}^{t} f(x)=f\left(x+t e_{i}\right)$. It follows from (20) that

$$
\nabla_{i} P_{j}(D)=\left(E_{i}^{1} P_{j}\right)(D) \nabla_{i} .
$$

Using (21) we compute the commutator of the operators $W_{i}, W_{j}$ :

$$
\begin{aligned}
W_{i} W_{j}-W_{j} W_{i}=( & \left.P_{i}(D)\left(E_{i}^{1} P_{j}\right)(D)-P_{j}(D)\left(E_{j}^{1} P_{i}\right)(D)\right) \nabla_{i} \nabla_{j} \\
& +\left(\left(E_{j}^{1} Q_{i}\right)(D)-Q_{i}(D)\right) P_{j}(D) \nabla_{j} \\
& +\left(Q_{j}(D)-\left(E_{i}^{1} Q_{j}\right)(D)\right) P_{i}(D) \nabla_{i} .
\end{aligned}
$$

Let us define the grade $g\left(x^{\alpha} z^{\beta}\right)$ of an element $x^{\alpha} z^{\beta}$ of the ring $R[x]$ to be $\alpha-\beta$. Notice that $g\left(D_{i}\left(x^{\alpha} z^{\beta}\right)\right)=\alpha-\beta$ and that $g\left(\nabla_{i}\left(x^{\alpha} z^{\beta}\right)\right)=\alpha-\beta+e_{i}$, for any 
$\alpha, \beta \in \mathrm{N}_{0}^{n}$. The result of the action of the operator in the right-hand side of (22) on $x^{\alpha} z^{\beta}$ consists of three terms whose grades are $\alpha-\beta+e_{i}+e_{j}, \alpha-\beta+e_{j}$ and $\alpha-\beta+e_{i}$. Thus the operators $W_{i}, W_{j}$ commute if and only if

$$
Q_{i}(D)=\left(E_{j}^{1} Q_{i}\right)(D), \quad i, j=1, \ldots, n, \quad i \neq j,
$$

and

$$
P_{i}(D)\left(E_{i}^{1} P_{j}\right)(D)=P_{j}(D)\left(E_{j}^{1} P_{i}\right)(D), \quad i, j=1, \ldots, n .
$$

The condition (23) is satisfied if and only if for each $i=1, \ldots, n$ the polynomial $Q_{i}(s)$ depends on $s_{i}$ only. Indeed, a polynomial is periodic with respect to one of its arguments if and only if it does not depend on this argument. Under this assumption on the polynomials $Q_{i}(s)$ the compatibility conditions (5) can be written in the form

$$
P_{i}\left(s+e_{j}\right) P_{j}(s)=P_{j}\left(s+e_{i}\right) P_{i}(s), \quad i, j=1, \ldots, n
$$

and they are therefore equivalent to (24). Thus the family of the operators $\left\{W_{i}\right\}_{i=1}^{n}$ is commutative if and only if the polynomials $P_{i}(s), Q_{i}(s)$ satisfy the compatibility conditions (5) and for each $i=1, \ldots, n$ the polynomial $Q_{i}(s)$ depends on $s_{i}$ only. The proof is complete.

For $x^{(0)} \in \mathrm{C}^{n}$ let $\hat{\mathscr{O}}_{x^{(0)}}$ be the $\mathscr{D}$-module of formal power series centered at $x^{(0)}$. Let $\mathrm{C}_{x^{(0)}}$ denote the set of complex numbers $\mathrm{C}$ considered as a $\mathrm{C}\left[x_{1}, \ldots\right.$, $\left.x_{n}\right]$-module via the isomorphism $\mathrm{C} \simeq \mathrm{C}\left[x_{1}, \ldots, x_{n}\right] /\left(x_{1}-x_{1}^{(0)}, \ldots, x_{n}-x_{n}^{(0)}\right)$. We use the following isomorphism (see Proposition 2.5.26 in [4] or [1], §4) between the space of formal solutions to $\mathscr{M}$ at $x^{(0)}$ and the dual space of $\mathrm{C}_{x^{(0)}} \otimes_{\mathrm{C}[x]} \mathscr{M}$

$$
\operatorname{Hom}_{\mathscr{D}}\left(\mathscr{M}, \hat{\mathscr{O}}_{x^{(0)}}\right) \simeq \operatorname{Hom}_{\mathrm{C}}\left(\mathrm{C}_{x^{(0)}} \otimes_{\mathrm{C}[x]} \mathscr{M}, \mathrm{C}\right) .
$$

This isomorphism holds for any finitely generated $\mathscr{D}$-module. Using (18) and fixing the point $x=x^{(0)}$ we arrive at the isomorphism

$$
\mathrm{C}_{x^{(0)}} \otimes_{\mathrm{C}[x]}\left(R[x] / \sum_{i=1}^{n} W_{i} R[x]\right) \simeq R / \sum_{i=1}^{n} W_{i, x^{(0)}} R,
$$

where $W_{i, x^{(0)}}$ are obtained from the operators $W_{i}$ by setting $x=x^{(0)}$. Combining (25) with (26) we see that

$$
\operatorname{Hom}_{\mathscr{D}}\left(\mathscr{M}, \hat{\mathscr{O}}_{x^{(0)}}\right) \simeq \operatorname{Hom}_{\mathrm{C}}\left(R / \sum_{i=1}^{n} W_{i, x^{(0)}} R, \mathrm{C}\right) .
$$


Thus the following Lemma holds true.

LEMma 2.6. The number of linearly independent formal power series solutions to the system (1) at the point $x=x^{(0)}$ is equal to $\operatorname{dim}_{\mathrm{C}} R / \sum_{i=1}^{n} W_{i, x^{(0)}} R$.

The following Lemma is motivated by the proof of Theorem 5.4 in [1].

Lemma 2.7. Let $L_{i}: R \rightarrow R, i=1, \ldots, n$ be a commutative family of linear operators such that there exists a regular sequence of homogeneous polynomials $f_{1}, \ldots, f_{n}$ in $R$ with the property $L_{i}(u)=f_{i} u+\tilde{u}$, where $\operatorname{deg} \tilde{u}<$ $\operatorname{deg}\left(f_{i} u\right)$. Then $R / \sum_{i=1}^{n} L_{i} R$ and $R /\left(f_{1}, \ldots, f_{n}\right)$ are isomorphic as C-vector spaces. Here $\left(f_{1}, \ldots, f_{n}\right)$ is the ideal generated by $f_{1}, \ldots, f_{n}$.

Proof. Let $\left\{u_{\alpha}\right\}_{\alpha \in \Lambda}$ represent a C-basis in $R /\left(f_{1}, \ldots, f_{n}\right)$ consisting of homogeneous polynomials. Let $u \in R, \operatorname{deg} u=k$, and let $X$ denote the set of all linear combinations of $\left\{u_{\alpha}\right\}_{\alpha \in \Lambda}$. We use induction on $k$ to show that $u \in X+\sum_{i=1}^{n} L_{i} R$. Since $u=\sum_{i=1}^{n} f_{i} v_{i}+\sum_{\alpha} c_{\alpha} u_{\alpha}$ for some $v_{1}, \ldots, v_{n} \in R$ and $c_{\alpha} \in \mathrm{C}$ it follows that

$$
u-\sum_{i=1}^{n} L_{i}\left(v_{i}\right)=u-\sum_{i=1}^{n} f_{i} v_{i}-\sum_{i=1}^{n} \tilde{v}_{i}=\sum_{\alpha} c_{\alpha} u_{\alpha}-\sum_{i=1}^{n} \tilde{v}_{i},
$$

where $\operatorname{deg}\left(\sum_{i=1}^{n} \tilde{v}_{i}\right)<k$. By induction $\sum_{i=1}^{n} \tilde{v}_{i} \in X+\sum_{i=1}^{n} L_{i} R$ which shows that $u \in X+\sum_{i=1}^{n} L_{i} R$. Thus $\left\{u_{\alpha}\right\}_{\alpha \in \Lambda}$ represents a generating set for the quotient $R / \sum_{i=1}^{n} L_{i} R$.

Let us show that $\left\{u_{\alpha}\right\}_{\alpha \in \Lambda}$ represent a set of linearly independent elements in $R / \sum_{i=1}^{n} L_{i} R$. Let $w \in X$ and suppose that $w=\sum_{i=1}^{n} L_{i} v_{i}$ for some $v_{i} \in R$. We show by induction on $k=\max _{i=1, \ldots, n} \operatorname{deg}\left(f_{i} v_{i}\right)$ that $w=0$. Let $v_{i}=$ $v_{i}^{\prime}+v_{i}^{\prime \prime}$, where $\operatorname{deg}\left(f_{i} v_{i}^{\prime}\right)=k$ and $\operatorname{deg}\left(f_{i} v_{i}^{\prime \prime}\right)<k$. Let $w_{k}$ be the homogeneous part of $w$ of degree $k$. Since $\left\{u_{\alpha}\right\}_{\alpha \in \Lambda}$ are homogeneous it follows that $w_{k} \in X$. Using the equality $w=\sum_{i=1}^{n} L_{i} v_{i}$ we obtain $w_{k}=\sum_{i=1}^{n} f_{i} v_{i}^{\prime}$. Since $\left\{u_{\alpha}\right\}_{\alpha \in \Lambda}$ represent a basis in $R /\left(f_{1}, \ldots, f_{n}\right)$ we have $X \cap \sum_{i=1}^{n} L_{i} R_{i}=\{0\}$. This shows that $\sum_{i=1}^{n} f_{i} v_{i}^{\prime}=0$. By the regularity of the sequence $\left(f_{1}, \ldots, f_{n}\right)$ there exists a skew-symmetric set $\left\{\eta_{i j}\right\}_{i, j=1}^{n}$ of homogeneous polynomials such that $v_{i}^{\prime}=\sum_{j=1}^{n} \eta_{i j} f_{j}$. Let us consider $\tilde{v}_{i}=v_{i}-\sum_{j=1}^{n} L_{j}\left(\eta_{i j}\right)$. Since the family of the operators $\left\{L_{i}\right\}_{i=1}^{n}$ is commutative it follows that $\sum_{i, j=1}^{n} L_{i} L_{j}\left(\eta_{i j}\right)=0$ and hence

$$
w=\sum_{i=1}^{n} L_{i}\left(v_{i}\right)=\sum_{i=1}^{n} L_{i}\left(\tilde{v}_{i}+\sum_{j=1}^{n} L_{j}\left(\eta_{i j}\right)\right)=\sum_{i=1}^{n} L_{i}\left(\tilde{v}_{i}\right) .
$$

Since $f_{i} \sum_{j=1}^{n} L_{j}\left(\eta_{i j}\right)$ and $f_{i} \sum_{j=1}^{n} \eta_{i j} f_{j}$ are equal up to the terms of degree less than $k$ it follows that $\operatorname{deg} f_{i} \tilde{v}_{i}<k$. By induction on $k$ we conclude that $w=0$, which completes the proof of the Lemma. 
For any differential operator $P \in \mathscr{D}, P=\sum_{|\alpha| \leq m} c_{\alpha}(x)\left(\frac{\partial}{\partial x}\right)^{\alpha}$ its principal symbol $\sigma(P)(x, z) \in R[x]$ is defined by $\sigma(P)(x, z)=\sum_{|\alpha|=m} c_{\alpha}(x) z^{\alpha}$. Let $H_{i}(x, z)=\sigma\left(G_{i}\right)(x, z)$ be the principal symbols of the differential operators which define the Horn system (1). Let $J \subset \mathscr{D}$ be the left ideal generated by $G_{1}, \ldots, G_{n}$. By the definition (see [3], Chapter $5, \S 2$ ) the characteristic variety $\operatorname{char}(\mathscr{M})$ of the Horn system is given by

$$
\operatorname{char}(\mathscr{M})=\left\{(x, z) \in \mathrm{C}^{2 n}: \sigma(P)(x, z)=0, \text { for all } P \in J\right\} .
$$

Let us define the set $U_{\mathscr{M}} \subset \mathrm{C}^{n}$ by $U_{\mathscr{M}}=\left\{x \in \mathrm{C}^{n}: \exists z \neq 0\right.$ such that $(x, z) \in$ $\operatorname{char}(\mathscr{M})\}$. Theorem 7.1 in [3, Chapter 5] yields that for $x^{(0)} \notin U_{\mathscr{M}}$

$$
\operatorname{Hom}_{\mathscr{D}}\left(\mathscr{M}, \hat{\mathscr{O}}_{x^{(0)}}\right) \simeq \operatorname{Hom}_{\mathscr{D}}\left(\mathscr{M}, \mathscr{O}_{x^{(0)}}\right) .
$$

It follows from [14, pages 148,146] that the C-dimension of the factor of the ring $R$ with respect to the ideal generated by the regular sequence of homogeneous polynomials $H_{1}\left(x^{(0)}, z\right), \ldots, H_{n}\left(x^{(0)}, z\right)$ is equal to the product $\prod_{i=1}^{n} \operatorname{deg} H_{i}\left(x^{(0)}, z\right)$. Since a sequence of $n$ homogeneous polynomials in $n$ variables is regular if and only if their common zero is the origin, it follows that $x^{(0)} \notin U_{\mathscr{M}}$ as long as the sequence $H_{1}\left(x^{(0)}, z\right), \ldots, H_{n}\left(x^{(0)}, z\right)$ is regular. Using Lemmas 2.5, 2.6 and 2.7 we arrive at the following Theorem.

Theorem 2.8. Suppose that the polynomials $P_{i}(s), Q_{i}(s)$ satisfy the compatibility conditions (5) and that for any $i=1, \ldots, n$ the polynomial $Q_{i}(s)$ depends on $s_{i}$ only. If the principal symbols $H_{1}(x, z), \ldots, H_{n}(x, z)$ of the differential operators $G_{1}, \ldots, G_{n}$ form a regular sequence at ${ }^{(0)}$ then the dimension of the space of holomorphic solutions to (1) at the point $x^{(0)}$ is equal to $\prod_{i=1}^{n} \operatorname{deg} H_{i}\left(x^{(0)}, z\right)$.

In the next section we, using Theorem 2.8, construct an explicit basis in the space of holomorphic solutions to the Horn system in the case when $\operatorname{deg} Q_{i}>$ $\operatorname{deg} P_{i}, i=1, \ldots, n$.

\section{Explicit basis in the solution space of some hypergeometric systems}

Suppose that the polynomials $P_{i}(s), Q_{i}(s)$ which determine the Horn system (1) satisfy the following conditions: for any $i=1, \ldots, n$ the polynomial $Q_{i}(s)$ depends on the variable $s_{i}$ only and $\operatorname{deg} Q_{i}>\operatorname{deg} P_{i}$. In this section we will show how to construct an explicit basis in the solution space of such a system of partial differential equations under some additional assumptions which are always satisfied if the parameters of the system under study are sufficiently general. 
Under the above conditions the polynomials $Q_{i}(s)$ can be represented in the form

$$
Q_{i}(s)=\prod_{j=1}^{d_{i}}\left(s_{i}-\alpha_{i j}\right), \quad i=1, \ldots, n, \quad \alpha_{i j} \in \mathrm{C} .
$$

By the Ore-Sato theorem [12] the general solution to the system of difference equations associated with (1) can be written in the form

$$
\varphi(s)=t_{1}^{s_{1}} \ldots t_{n}^{s_{n}} \frac{\prod_{i=1}^{p} \Gamma\left(\left\langle A_{i}, s\right\rangle-c_{i}\right)}{\prod_{i=1}^{n} \prod_{j=1}^{d_{i}} \Gamma\left(s_{i}-\alpha_{i j}+1\right)} \phi(s),
$$

where $p \in \mathrm{N}_{0}, d_{i}=\operatorname{deg} Q_{i}, t_{i}, c_{i} \in \mathrm{C}, A_{i} \in \mathrm{Z}^{n}$ and $\phi(s)$ is an arbitrary periodic function with the period 1 in each variable. The following Theorem holds true.

THEOREM 3.1. Suppose that the following conditions are fulfilled.

1. For any $i=1, \ldots, n$ the polynomial $Q_{i}(s)$ depends on the variable $s_{i}$ only and $\operatorname{deg} Q_{i}>\operatorname{deg} P_{i}$.

2. The difference $\alpha_{i j}-\alpha_{i k}$ is never equal to a real integer number, for any $i=1, \ldots, n$ and $j \neq k$.

3. For any multi-index $I=\left(i_{1}, \ldots, i_{n}\right)$ with $i_{k} \in\left\{1, \ldots, d_{k}\right\}$ the product $\prod_{i=1}^{p}\left(\left\langle A_{i}, s\right\rangle-c_{i}\right)$ never vanishes on the shifted lattice $Z^{n}+\gamma_{I}$, where $\gamma_{I}=$ $\left(\alpha_{1 i_{1}}, \ldots, \alpha_{n i_{n}}\right)$.

Then the family consisting of $\prod_{i=1}^{n} d_{i}$ functions

$$
y_{I}(x)=x^{\gamma_{I}} \sum_{s \in N_{0}^{n}} t^{s+\gamma_{I}} \frac{\prod_{i=1}^{p} \Gamma\left(\left\langle A_{i}, s+\gamma_{I}\right\rangle-c_{i}\right)}{\prod_{k=1}^{n} \prod_{j=1}^{d_{k}} \Gamma\left(s_{k}+\alpha_{k i_{k}}-\alpha_{k j}+1\right)} x^{s}
$$

is a basis in the space of holomorphic solutions to the system (1) at any point $x \in\left(\mathrm{C}^{*}\right)^{n}=(\mathrm{C} \backslash\{0\})^{n}$.

Proof. It follows from Theorem 1.3 and the assumptions 2,3 of Theorem 3.1 that the series (27) formally satisfies the Horn system (1). Since $\operatorname{deg} Q_{i}(s)>\operatorname{deg} P_{i}(s), i=1, \ldots, n$ it follows by the construction of the function (6) (see [10]) that all the components of the vector $\triangle=\sum_{i=1}^{p} A_{i}-$ $\left(d_{1}, \ldots, d_{n}\right)$ are negative. Thus for any multi-index $I$ the intersection of the half-space $\operatorname{Re}\langle\triangle, s\rangle \geq 0$ with the shifted positive octant $\mathrm{R}_{+}^{n}+\gamma_{I}$ is a bounded set. Using the Stirling formula we conclude that the series (27) converges everywhere in $\left(\mathrm{C}^{*}\right)^{n}$ for any multi-index $I$ (see also $\$ 2.4$ in [11]). The set $\left\{x \in \mathrm{C}^{n}: x_{1} \ldots x_{n}=0\right\}$ is special since (27) can contain terms with negative powers of $x$. 
The series (27) corresponding to different multi-indices $I, J$ are linearly independent since by the second assumption of Theorem 3.1 their initial monomials $x^{\gamma_{I}}, x^{\gamma_{J}}$ are different. Finally, the conditions of Theorem 2.8 are satisfied in our setting since the first assumption of Theorem 3.1 yields that the sequence of principal symbols $H_{1}\left(x^{(0)}, z\right), \ldots, H_{n}\left(x^{(0)}, z\right) \in R$ of hypergeometric differential operators defining the Horn system is regular for $x^{(0)} \in\left(C^{*}\right)^{n}$. Indeed, in our case $H_{i}\left(x^{(0)}, z\right)=\left(x_{i}^{(0)}\right)^{d_{i}} z_{i}^{d_{i}}$. Hence by Theorem 2.8 the number of linearly independent holomorphic solutions to the system under study at a generic point equals $\prod_{i=1}^{n} d_{i}$. In this case $U_{\mathscr{M}}=\left\{x^{(0)} \in \mathrm{C}^{n}: x_{1}^{(0)} \ldots x_{n}^{(0)}=0\right\}$. Thus the series (27) span the space of holomorphic solutions to the system (1) at any point $x^{(0)} \in\left(\mathrm{C}^{*}\right)^{n}$. The proof is complete.

REMARK 3.2. The fact that the dimension of the solution space of (1) at a generic point is equal to the product of the degrees of the polynomials $Q_{1}(s), \ldots, Q_{n}(s)$ in the case when $\operatorname{deg} Q_{i}>\operatorname{deg} P_{i}, i=1, \ldots, n$ follows also from Theorem 5.1.1 in [9]. Theorem 3.1 gives an explicit description of the solutions to the Horn system under two additional assumptions 2 and 3 which are always satisfied if the parameters of the given system are sufficiently general.

In the theory developed by Gelfand, Kapranov and Zelevinsky the conditions 2 and 3 of Theorem 3.1 correspond to the so-called nonresonant case (see [6], §8.1). Thus the result on the structure of solutions to the Horn system can be formulated as follows.

Corollary 3.3. Let $x^{(0)} \in\left(C^{*}\right)^{n}$ and suppose that for any $i=1, \ldots, n$ the polynomial $Q_{i}(s)$ depends on the variable $s_{i}$ only and that $\operatorname{deg} Q_{i}>\operatorname{deg} P_{i}$. If the parameters of the system (1) are nonresonant then there exists a basis in the space of holomorphic solutions to (1) near $x^{(0)}$ whose elements are given by series of the form (2).

Let us now consider some examples. We begin with a simple example of a system of the first order.

Example 3.4. Consider the following system of the Horn type

$$
\left\{\begin{array}{l}
x_{1}\left(x_{1} \frac{\partial}{\partial x_{1}}+x_{2} \frac{\partial}{\partial x_{2}}+2\right) y(x)=\left(x_{1} \frac{\partial}{\partial x_{1}}\right) y(x), \\
x_{2}\left(x_{1} \frac{\partial}{\partial x_{1}}+x_{2} \frac{\partial}{\partial x_{2}}+2\right) y(x)=\left(x_{2} \frac{\partial}{\partial x_{2}}\right) y(x) .
\end{array}\right.
$$

The principal symbols $H_{1}(x, z), H_{2}(x, z) \in R[x]$ of the differential operators 
defining the system (28) are as follows

$$
H_{1}(x, z)=\left(x_{1}^{2}-x_{1}\right) z_{1}+x_{1} x_{2} z_{2}, \quad H_{2}(x, z)=x_{1} x_{2} z_{1}+\left(x_{2}^{2}-x_{2}\right) z_{2} .
$$

By Theorem 2.8 the dimension of the solution space of (28) at a generic point $x \in \mathrm{C}^{2}$ is equal to $\operatorname{dim}_{\mathrm{C}} \mathrm{C}\left[z_{1}, z_{2}\right] /\left(H_{1}(x, z), H_{2}(x, z)\right)$. This dimension is equal to 1 whenever the determinant

$$
\left|\begin{array}{cc}
x_{1}^{2}-x_{1} & x_{1} x_{2} \\
x_{1} x_{2} & x_{2}^{2}-x_{2}
\end{array}\right|=x_{1} x_{2}\left(1-x_{1}-x_{2}\right)
$$

is nonzero. Thus the dimension of the solution space of (28) at a generic point equals 1.

Let us find the solution to (28). We may choose $\gamma=0$ in this example. The system of difference equations which corresponds to the system (28) is as follows

$$
\left\{\begin{array}{l}
\varphi\left(s+e_{1}\right)\left(s_{1}+1\right)=\varphi(s)\left(s_{1}+s_{2}+2\right), \\
\varphi\left(s+e_{2}\right)\left(s_{2}+1\right)=\varphi(s)\left(s_{1}+s_{2}+2\right)
\end{array}\right.
$$

Let us consider (29) for $s$ being a continuous argument varying over $\mathrm{C}^{2}$. By Theorem 1.1 the general solution to (29) is given by

$$
\varphi(s)=\frac{\Gamma\left(s_{1}+s_{2}+2\right)}{\Gamma\left(s_{1}+1\right) \Gamma\left(s_{2}+1\right)} \phi(s),
$$

where $\phi(s)$ is an arbitrary periodic function with the period 1 in $s_{1}$ and $s_{2}$. (It is straightforward to check that (30) is indeed a solution to (29); Theorem 1.1 yields that this solution is general since any two solutions differ at most in periodic factors.) We use (30) for constructing all solutions to (29) in the case when $s \in Z^{2}$. There exist three $Z^{2}$-connected subsets of the lattice $Z^{2}$ which satisfy the conditions of Theorem 1.3, namely

$$
\begin{aligned}
& S_{1}=\left\{\left(s_{1}, s_{2}\right) \in Z^{2}: s_{1} \geq 0, s_{2} \geq 0\right\}, \\
& S_{2}=\left\{\left(s_{1}, s_{2}\right) \in Z^{2}: s_{1} \geq 0, s_{1}+s_{2}+2 \leq 0\right\}, \\
& S_{3}=\left\{\left(s_{1}, s_{2}\right) \in Z^{2}: s_{2} \geq 0, s_{1}+s_{2}+2 \leq 0\right\} .
\end{aligned}
$$

These irreducible supports of solutions to (29) are shown in Figure 1. 


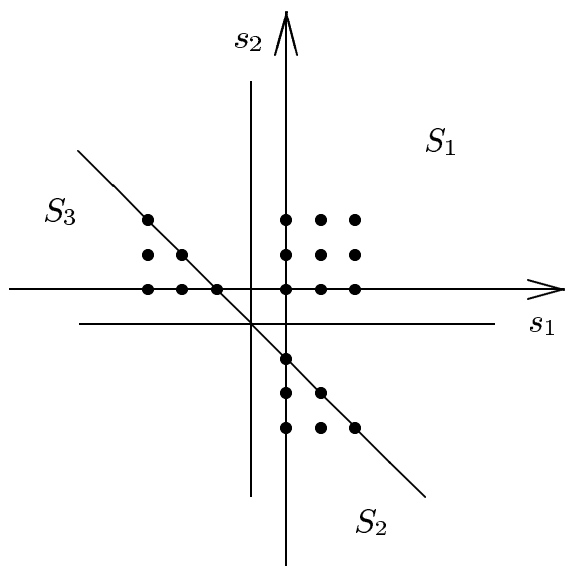

FigURE 1 .

However, the power series which correspond to these irreducible supports give the same solution to (28) since they represent the same meromorphic function in different domains. Indeed, choosing $\phi(s) \equiv 1$ on $S_{1}$ we obtain a solution to (28):

$$
y_{1}(x)=\sum_{s_{1}, s_{2} \geq 0} \frac{\Gamma\left(s_{1}+s_{2}+2\right)}{\Gamma\left(s_{1}+1\right) \Gamma\left(s_{2}+1\right)} x_{1}^{s_{1}} x_{2}^{s_{2}}=\frac{1}{\left(1-x_{1}-x_{2}\right)^{2}} .
$$

We have to choose the periodic function $\phi(s)$ in a nontrivial way (as it was explained in section 1) in order to check that the series which correspond to $S_{2}, S_{3}$ converge to the same function. Choosing $\phi(s)=\left((-1)^{s_{1}} \sin \pi\left(s_{1}+\right.\right.$ $\left.\left.s_{2}\right)\right) / \sin \pi\left(s_{2}+1\right)$ and using the Euler completion formula $\Gamma(z) \Gamma(1-z)=$ $\pi / \sin \pi z$ we obtain the series

$$
y_{2}(x)=\sum_{\substack{s_{1} \geq 0, s_{1}+s_{2}+2 \leq 0}} \frac{(-1)^{s_{1}} \Gamma\left(-s_{2}\right)}{\Gamma\left(s_{1}+1\right) \Gamma\left(-s_{1}-s_{2}-1\right)} x_{1}^{s_{1}} x_{2}^{s_{2}}=\frac{1}{\left(1-x_{1}-x_{2}\right)^{2}} .
$$

Analogously, choosing $\phi(s)=\left((-1)^{s_{2}} \sin \pi\left(s_{1}+s_{2}\right)\right) / \sin \pi\left(s_{1}+1\right)$ we arrive at the series

$$
y_{3}(x)=\sum_{\substack{s_{2} \geq 0, s_{1}+s_{2}+2 \leq 0}} \frac{(-1)^{s_{2}} \Gamma\left(-s_{1}\right)}{\Gamma\left(s_{2}+1\right) \Gamma\left(-s_{1}-s_{2}-1\right)} x_{1}^{s_{1}} x_{2}^{s_{2}}=\frac{1}{\left(1-x_{1}-x_{2}\right)^{2}} .
$$

Thus the series which correspond to the irreducible supports $S_{1}, S_{2}, S_{3}$ constitute analytic continuations of one another. 
It is easy to check that the space of holomorphic solutions to (28) at a point $x \in C^{2}$ such that $1-x_{1}-x_{2} \neq 0$ is indeed spanned by the function $\left(1-x_{1}-x_{2}\right)^{-2}$. The equations (28) yield that $y_{x_{1}}^{\prime}=y_{x_{2}}^{\prime}$ and hence $y\left(x_{1}, x_{2}\right)=u\left(x_{1}+x_{2}\right)$ for some differentiable function $u(t)$. Solving the corresponding ordinary differential equation of the first order we conclude that any holomorphic solution to (28) is a multiple of the function (31).

ExAmPLe 3.5. Let us consider the following system of hypergeometric differential equations of the Horn type

$$
\left\{\begin{array}{r}
x_{1}\left(x_{1} \frac{\partial}{\partial x_{1}}+x_{2} \frac{\partial}{\partial x_{2}}-4\right)\left(x_{1} \frac{\partial}{\partial x_{1}}+1\right) y(x) \\
=\left(x_{1} \frac{\partial}{\partial x_{1}}-3\right)\left(x_{1} \frac{\partial}{\partial x_{1}}-1\right) y(x), \\
x_{2}\left(x_{1} \frac{\partial}{\partial x_{1}}+x_{2} \frac{\partial}{\partial x_{2}}-4\right)\left(x_{2} \frac{\partial}{\partial x_{2}}+2\right) y(x) \\
=\left(x_{2} \frac{\partial}{\partial x_{2}}-3\right)\left(x_{2} \frac{\partial}{\partial x_{2}}-1\right) y(x) .
\end{array}\right.
$$

The principal symbols $H_{1}(x, z), H_{2}(x, z) \in R[x]$ of the differential operators defining the system (32) are as follows

$$
H_{1}(x, z)=x_{1}^{2} z_{1}\left(\left(x_{1}-1\right) z_{1}+x_{2} z_{2}\right), \quad H_{2}(x, z)=x_{2}^{2} z_{2}\left(x_{1} z_{1}+\left(x_{2}-1\right) z_{2}\right) .
$$

By Theorem 2.8 the dimension of the solution space of the system (32) is equal to 4 everywhere except for the set $\left\{\left(x_{1}, x_{2}\right) \in \mathrm{C}^{2}: x_{1} x_{2}\left(1-x_{1}\right)\left(1-x_{2}\right)(1-\right.$ $\left.\left.x_{1}-x_{2}\right)=0\right\}$ on which the sequence $H_{1}(x, z), H_{2}(x, z)$ is not regular. To obtain the solutions to (32) we let $\gamma=0$ and consider the associated system of difference equations

$$
\left\{\begin{array}{l}
\varphi\left(s+e_{1}\right) s_{1}\left(s_{1}-2\right)=\varphi(s)\left(s_{1}+s_{2}-4\right)\left(s_{1}+1\right), \\
\varphi\left(s+e_{2}\right) s_{2}\left(s_{2}-2\right)=\varphi(s)\left(s_{1}+s_{2}-4\right)\left(s_{2}+2\right) .
\end{array}\right.
$$

Let us consider (33) for $s$ being a continuous argument varying over $\mathrm{C}^{2}$. By Theorem 1.1 the general solution to the system (33) is given by

$$
\varphi(s)=\frac{\Gamma\left(s_{1}+s_{2}-4\right)}{\Gamma\left(s_{1}-2\right) \Gamma\left(s_{2}-2\right)} s_{1} s_{2}\left(s_{2}+1\right) \phi(s),
$$

where $\phi(s)$ is an arbitrary periodic function with the period 1 in $s_{1}$ and $s_{2}$. In this example there exist six different $Z^{2}$-connected subsets of the lattice $Z^{2}$ 
which satisfy the conditions of Theorem 1.3, namely

$$
\begin{aligned}
& S_{1}=\left\{\left(s_{1}, s_{2}\right) \in Z^{2}: s_{1}=3, s_{2}=1\right\}, \\
& S_{2}=\left\{\left(s_{1}, s_{2}\right) \in Z^{2}: s_{1}=1, s_{2}=3\right\}, \\
& S_{3}=\left\{\left(s_{1}, s_{2}\right) \in Z^{2}: 3 \leq s_{1}, 3 \leq s_{2}\right\}, \\
& S_{4}=\left\{\left(s_{1}, s_{2}\right) \in Z^{2}: s_{1} \leq-1, s_{2} \leq-2\right\}, \\
& S_{5}=\left\{\left(s_{1}, s_{2}\right) \in Z^{2}: s_{1} \leq-1,3 \leq s_{2}, s_{1}+s_{2} \leq 4\right\}, \\
& S_{6}=\left\{\left(s_{1}, s_{2}\right) \in Z^{2}: 3 \leq s_{1}, s_{2} \leq-2, s_{1}+s_{2} \leq 4\right\} .
\end{aligned}
$$

These irreducible supports of solutions to (33) are displayed in Figure 2.

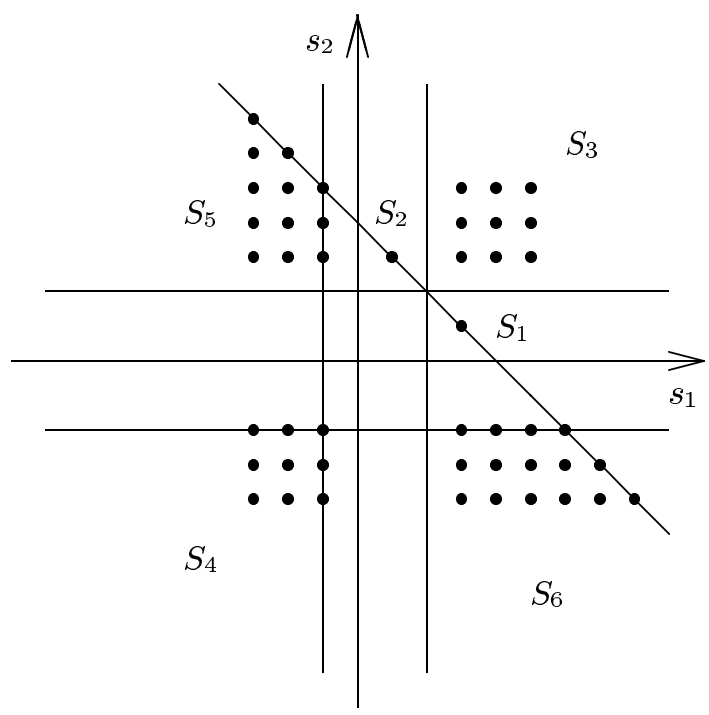

FiguRE 2.

Since two of the irreducible supports are finite and each one of them consists of one point it follows that the given system has two monomial solutions:

$$
y_{1}(x)=x_{1}^{3} x_{2}, \quad y_{2}(x)=x_{1} x_{2}^{3} .
$$

Choosing the periodic function $\phi(s)$ as it was explained in section 1 and computing the sum of the series supported in $S_{3}$ we obtain the third solution to the system (32):

$$
\begin{aligned}
y_{3}(x)=x_{1}^{3} x_{2}^{3} & \left(2 x_{1}^{3}+10 x_{1}^{2} x_{2}+5 x_{1} x_{2}^{2}+x_{2}^{3}\right. \\
& \left.-10 x_{1}^{2}-20 x_{1} x_{2}-5 x_{2}^{2}+14 x_{1}+10 x_{2}-6\right) /\left(1-x_{1}-x_{2}\right)^{5} .
\end{aligned}
$$


The sums of the series supported in the sets $S_{5}$ and $S_{6}$ can be expressed through $y_{1}(x), y_{2}(x), y_{3}(x)$. More precisely, the following linear relations hold:

$$
y_{5}(x)=2 y_{2}(x)+y_{3}(x), \quad y_{6}(x)=y_{1}(x)+y_{3}(x) .
$$

We choose $\phi(s)$ as in section 1 and compute the remaining solution to the system under study which is given by the series supported in $S_{4}$ :

$$
y_{4}(x)=\sum_{s_{1}, s_{2} \geq 0} \frac{\Gamma\left(s_{1}+4\right) \Gamma\left(s_{2}+5\right)}{\Gamma\left(s_{1}+s_{2}+8\right)}\left(s_{1}+1\right)\left(s_{2}+1\right)\left(s_{2}+2\right) x_{1}^{-s_{1}-1} x_{2}^{-s_{2}-2} .
$$

The sum of this series can be expressed through the Appel function $F_{3}$ (see [13], section 1.3):

$$
y_{4}(x)=\left.\frac{\Gamma(4) \Gamma(5)}{\Gamma(8)}\left(\xi_{1} \xi_{2}^{2} \frac{\partial}{\partial \xi_{1}} \frac{\partial^{2}}{\partial \xi_{2}^{2}}\left(\xi_{1} \xi_{2}^{2} F_{3}\left[4,5,1,1,8, \xi_{1}, \xi_{2}\right]\right)\right)\right|_{\substack{\xi_{1}=1 / x_{1} \\ \xi_{2}=1 / x_{2}}} .
$$

The constructed solutions $y_{1}(x), y_{2}(x), y_{3}(x), y_{4}(x)$ to the system (32) form a basis in its solution space at any point $x \in C^{2}$ such that $x_{1} x_{2}\left(1-x_{1}\right)(1-$ $\left.x_{2}\right)\left(1-x_{1}-x_{2}\right) \neq 0$.

\section{REFERENCES}

1. Adolphson, A., Hypergeometric functions and rings generated by monomials, Duke Math. J. 73 (1994), 269-290.

2. Aigner, M., Combinatorial Theory, Springer-Verlag, 1979.

3. Björk, J. -E., Rings of Differential Operators, North. Holland Mathematical Library, 1979.

4. Björk, J. -E., Analytic D D-Modules and Applications, Kluwer Academic Publishers, 1993.

5. Evgrafov, M. A., Series and Integral Representations, Progress in Science and Technology. Current Problems of Mathematics. Fundamental Directions, Moscow, 1986. Vol. 13.

6. Gelfand, I. M. and Graev, M. I., GG-functions and their relation to general hypergeometric functions, Russian Math. Surveys 52 (1997), 639-684.

7. Gelfand, I. M., Graev, M. I. and Retach, V. S., General hypergeometric systems of equations and series of hypergeometric type, Russian Math. Surveys 47 (1992), 1-88.

8. Horn, J., Über hypergeometrische Funktionen zweier Veränderlichen, Math. Ann. 117 (1940), 384-414.

9. Hörmander, L., Linear Partial Differential Operators, Springer-Verlag, 1963.

10. Sadykov, T. M., On a multidimensional system of hypergeometric differential equations, Siberian Math. J. 39 (1998), 986-997.

11. Sadykov, T. M., Systems of partial differential equations of hypergeometric type, Licentiate of philosophy thesis. Stockholm University, (1999), ISBN 91-7153-872-0.

12. Sato, M., Theory of prehomogeneous vector spaces (algebraic part), Nagoya Math. J. 120 (1990), 1-34.

13. Srivastava, H. M. and Karlsson, P. W., Multiple Gaussian Hypergeometric Series, Ellis Horwood Limited, 1985. 
14. Tsikh, A. K., Multidimensional Residues and Their Applications, Transl. Math. Monographs 103 (1992).

DEPARTMENT OF MATHEMATICS

UNIVERSITY OF STOCKHOLM

S-10691 STOCKHOLM

SWEDEN

E-mail: timur@matematik.su.se 